\title{
A new era for proteomics research? Mathias Uhlen
}

Address: School of Biotechnology, AlbaNova University Center, Royal Institute of Technology (KTH), Stockholm SE-10691, Sweden. Email: mathias@biotech.kth.se

Published: 4 November 2008

Genome Biology 2008, 9:325 (doi:10.1 186/gb-2008-9-11-325)

The electronic version of this article is the complete one and can be found online at http://genomebiology.com/2008/9/I I/325

(c) 2008 BioMed Central Ltd

A report of the 7th Annual Human Proteome Organization (HUPO) Conference, Amsterdam, the Netherlands, 16-20 August 2008.

The human genome sequence provided information on the protein-encoding genes that are expressed in the hundreds of cell types that make up the human body. The next step forward is to use the information from genomics research for a systematic study of the human proteome. At the recent Human Proteome Organization (HUPO) conference in Amsterdam, various approaches to such studies were discussed. Topics covered the use of proteomics to study biology in humans and model organisms, including investigations aimed at increasing the understanding of cellular pathways, the biology of stem cells, and subcellular organization.

The state of the art in mass spectrometry was addressed in several keynote lectures. Refined instrumentation and more advanced software for analysis means that whole-proteome coverage for model organisms in a single experiment can now be envisaged and relatively low-abundance mammalian proteins can be detected. Ruedi Aebersold (ETH, Zurich, Switzerland and ISB, Seattle, USA) described the identification of 'proteotypic peptides' - protein fragments that are detectable in mass spectrometry - and pointed out that a vision for the future might be to identify and publish such peptides for all human proteins and to make these publicly available through web portals such as the Peptide Atlas [www.peptideatlas.org]. Matthias Mann (Max Planck Institute of Biochemistry, Martinsried, Germany) focused on the advantages of using stable isotopes to achieve higher accuracy in quantitative measurements of proteins. He described the use of SILAC (stable isotope labeling with amino acids in cell culture) for the study of proteins in cell lines and model organisms such as rat and mouse. By combining kinase-specific affinity purification and quantitative mass spectrometry, more than 1,000 phosphorylation sites on human protein kinases were identified, and interestingly, more than half of these were upregulated during mitosis in human cancer cells.

\section{An atlas for human protein distribution}

The use of antibodies and other affinity reagents to study the human proteome was the topic of many talks. Emma Lundberg (Royal Institute of Technology, Stockholm, Sweden) described the use of antibodies from the Human Protein Atlas program [www.proteinatlas.org] to explore the subcellular localization of proteins in three human cell lines of glioma, epithelial and mesenchymal origin. An analysis of 2,000 proteins suggested that approximately one-third were localized mainly to the nucleus. Version 4.0 of the Human Protein Atlas was launched at the conference. This compendium of protein distribution now contains data from experiments with 6,000 antibodies and more than 5 million high-resolution images, double the content of the previous year. Profiles showing a protein's distribution in cells, tissues and organs cover more than 5,000 genes, approximately $25 \%$ of human protein-coding genes.

Erik Björling (Royal Institute of Technology, Stockholm, Sweden) presented a new gene-centric organization of the atlas, which now allows advanced queries involving protein classes, chromosomal location and protein profiles in normal and cancer tissues. Fredrik Ponten (Uppsala University, Sweden) described the use of the portal as a discovery tool for potential biomarkers in the fields of breast, colorectal, prostate and lung cancer. He presented several examples with prognostic value, such as the putative human transcription factor SATB2 for the prediction of outcome for colorectal cancer patients, as evaluated by subsequent analysis using validation cohorts containing many hundreds of disease-specific samples from patients.

\section{Proteome biology and interaction analysis}

It is evident that human biology depends on precise regulation of protein concentrations in space and time and 
intricate interactions between different protein isoforms to form stable complexes and transient interaction networks. This topic was addressed by Anne-Claude Gavin (EMBL, Heidelberg, Germany), who reviewed progress in the global analysis of biomolecular interactions. Genome-wide scans in model organisms have led to network maps of general relevance for eukaryotic organisms. The generation of comprehensive maps involving specific pathways can be used to identify potential therapeutic targets. Gavin pointed out that one of the challenges for the future is to adapt quantitative biochemical interaction analysis for proteome-wide efforts.

Tony Pawson (University of Toronto, Canada) is using a combination of classical cell biology techniques with quantitative mass spectrometry to study signaling pathways. In a study of the formation of tight junctions in kidney cells, he has found that the protein phosphatase PP1alpha binds to multiple sites on the Par-3 protein, regulating the binding of other proteins. The results suggest that Par- 3 acts as a scaffold for both serine/threonine kinases and PP1 phosphatase. Pawson's talk provided a good example of how the new generation of quantitative proteomics platforms can be used to provide detailed analysis of cell signaling based on temporal and spatial proteomics. Hans Clevers (Hubrecht Laboratory, Utrecht, the Netherlands) described a detailed analysis of the stem cells of the intestinal epithelium using a combination of molecular biology and proteomics. The intestinal epithelium is one of the most proliferative tissues in humans, making the intestinal crypt an interesting model for studies of adult stem cell biology. Clevers described the unique role of the LGR5 protein expressed specifically in the intestinal stem cells at the crypt bottom, based on a number of different approaches, including knock-in mouse experiments and antibody-based protein profiling.

\section{Outlook for a Human Proteome Project}

Amos Bairoch (Swiss Institute of Bioinformatics, Geneva, Switzerland), whose group administrates the SwissProt/ UniProt protein database, announced that the number of human protein-coding genes was currently estimated to be 20,400 , although this number is likely to change over the next few years. Interestingly, he reported that for almost 9,000 (44\%) of these genes, there is still no experimental information about the proteins they encode. This emphasizes the need for a systematic effort to characterize human proteins to leverage information from the genome project and to form a basis for further studies. At the conference, the possible launch of a Human Proteome Project to systematically map the proteome was discussed with representatives from funding agencies from Europe, USA, Canada and Asia. John Bergeron (McGill University, Montreal, Canada) proposed a gene-centric approach based on three technology platforms (mass spectrometry, antibodies and interaction analysis) to characterize at least one representative protein variant from every gene locus. Several participants pointed out the difficulty of a gene-centric approach for mass spectrometry, whereas such an approach is more feasible for antibody- and interaction-based analysis. The shortcomings of previous systematic proteomics efforts were brought up, emphasizing the need for increased precision of protein identification by mass spectrometry. Representatives of several funding agencies pointed out the importance of focused pilot projects to show the feasibility of the different parts of the effort, and emphasized the need for clear end-points and international coordination.

The conference demonstrated that proteomics research has entered a new era of biology-driven applications, with studies of signal pathways and protein profiles in various cells and tissues based on advances in the use of proteomics tools. The rapid development of mass-spectrometry based, immuno-based and gene-tagging technologies has opened up the possibility of whole-proteome analysis. The challenge for the proteomics community now is to show how the human proteome can be experimentally annotated, and to deliver a human proteome 'parts-list' with data on localization and function within a reasonable time-frame. Such a list, with its accompanying resources of protein-specific probes (antibodies), proteotypic peptides and cDNA clones, would provide a valuable asset for hypothesis-driven research into human biology and disease. Such efforts will no doubt be discussed at the next HUPO conference, to be held in Toronto, Canada, in September 2009. 